\title{
Alcohol Consumption and Night-Time Leisure among Brazilian University Students
}

- Heloisa Freitas,

- Susana Henriques,

- Ricardo R. Uvinha,

- Carolin Lusby \&

- Liana Romera

International Journal of the Sociology of Leisure (2020)

\begin{abstract}
Recreational drug use is recognized by the World Health Organization as the consumption of alcoholic beverages, carried out by a portion of youth during leisure contexts. This research aimed at knowing the types of recreational drug use of a population of young Brazilian university students. This is an exploratory and quantitative research, developed through an anonymous and semi-structured questionnaire. The sample consisted of 217 university students, aged 18 to 24 from a public university in the city of Vitoria- Brazil. The results showed that the recreational uses of substances occur mostly during leisure time. Such scenarios represent, for these young people, the possibility of rupture with daily life and the adult universe, characterized by the imposition of rules. For a young group, night-time leisure represents an important context of sociability and alcohol consumption. Among those surveyed, $63.6 \%$ consumed alcoholic beverages at night outings and $45.1 \%$ almost every weekend. Regarding the pattern of use, $49 \%$ consume binge drinking, especially when drinking in informal spaces such as streets and squares due to the lower regulatory control. The results corroborate that alcohol consumption is related to leisure agendas and address issues such as autonomous expression of identities and lifestyles in contemporary society.
\end{abstract}

